\title{
The effect of hydrophobic glassy organic material on the cloud condensa- tion nuclei activity of particles with different morphologies
}

\section{Ankit Tandon et al.}

Correspondence to: Markus D. Petters (markus_petters@ncsu.edu)

The copyright of individual parts of the supplement might differ from the CC BY 4.0 License. 


\section{Overview}

Section S1 contains supporting data for the CCN experiments, including the relationship between instrument nominal temperature gradient and instrument supersaturation and experiments used to constrain the CCN activity of polyethylene particles. Section S2 contains tabulated data. Tables S1-S8 summarize data needed to reproduce Figures 2-5 in the main manuscript and Figures S1-S2 in this document.

\section{Section S1. CCN Experiments}

Figure S1 shows the relationship between instrument nominal temperature gradient and instrument supersaturation from the ammonium sulfate calibration. The experiment was performed using a TSI 3080 long DMA column, a TSI 3771 condensation particle counter and the DMT CCN instrument operated at a flow rate of $0.3 \mathrm{~L} \mathrm{~min}^{-1}$. Note that the calibration was performed on a different DMA/CCN/CPC setup than the experiments performed in the main paper, which used the radial DMA and TSI 3020, which are used as detector for the coagulation instrument.

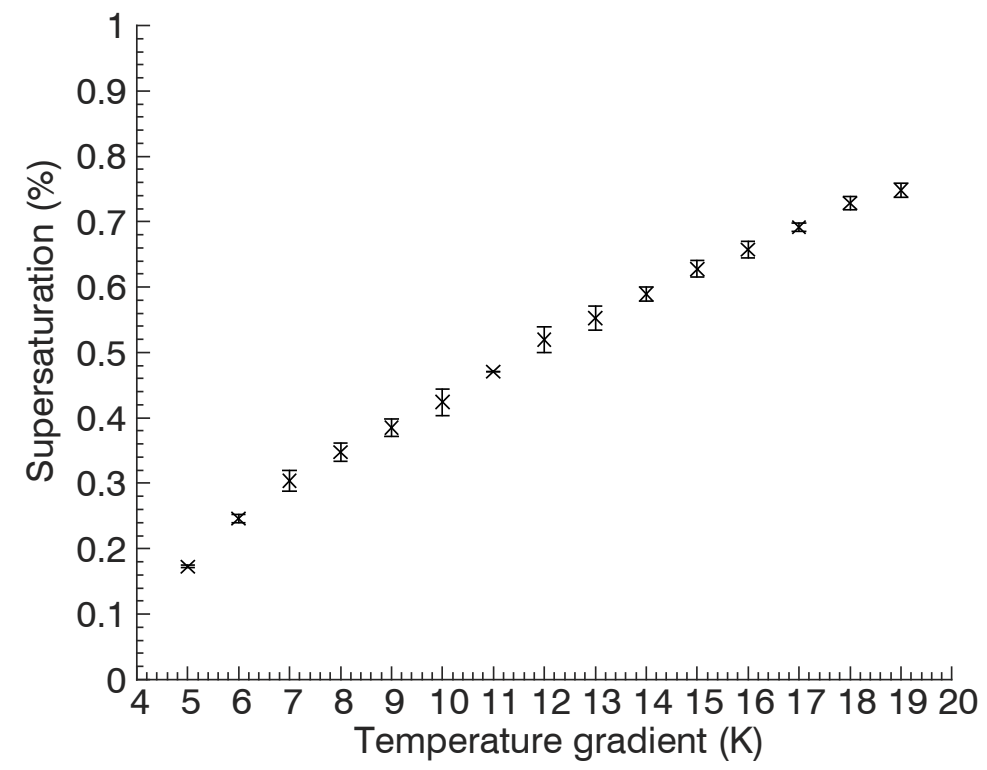

Figure S1. Relationship between instrument nominal temperature gradient and instrument supersaturation from the ammonium sulfate calibration at $\mathrm{CCNc}$ flow rate $0.3 \mathrm{~L} \mathrm{~min}^{-1}$. Vertical bars show the standard deviation of the inferred supersaturation.

Figure S2 summarizes the experiments used to constrain the CCN activity of polyethylene particles. Here the $\mathrm{CCNc}$ was operated at the highest achievable supersaturation corresponding to $\mathrm{dT}=20 \mathrm{~K}$ and a flow rate of $1 \mathrm{~L} \mathrm{~min}^{-1}$. Experiments were performed using a high-flow DMA column to extend the diameter range to $750 \mathrm{~nm}$. The concentration of $1 \mathrm{~cm}^{-3} \mathrm{CCN}$ particles corresponds to the noise floor of the measurement in this experiment. For the glucose experiment, the fraction of particles activating at $\mathrm{D}<100 \mathrm{~nm}$ is less than unity. This is due to losses in the inlet system. The activated fraction at $23.4 \mathrm{~nm}$ is 0.68 (Table S6); glucose particles of that size are still $\mathrm{CCN}$ active. 

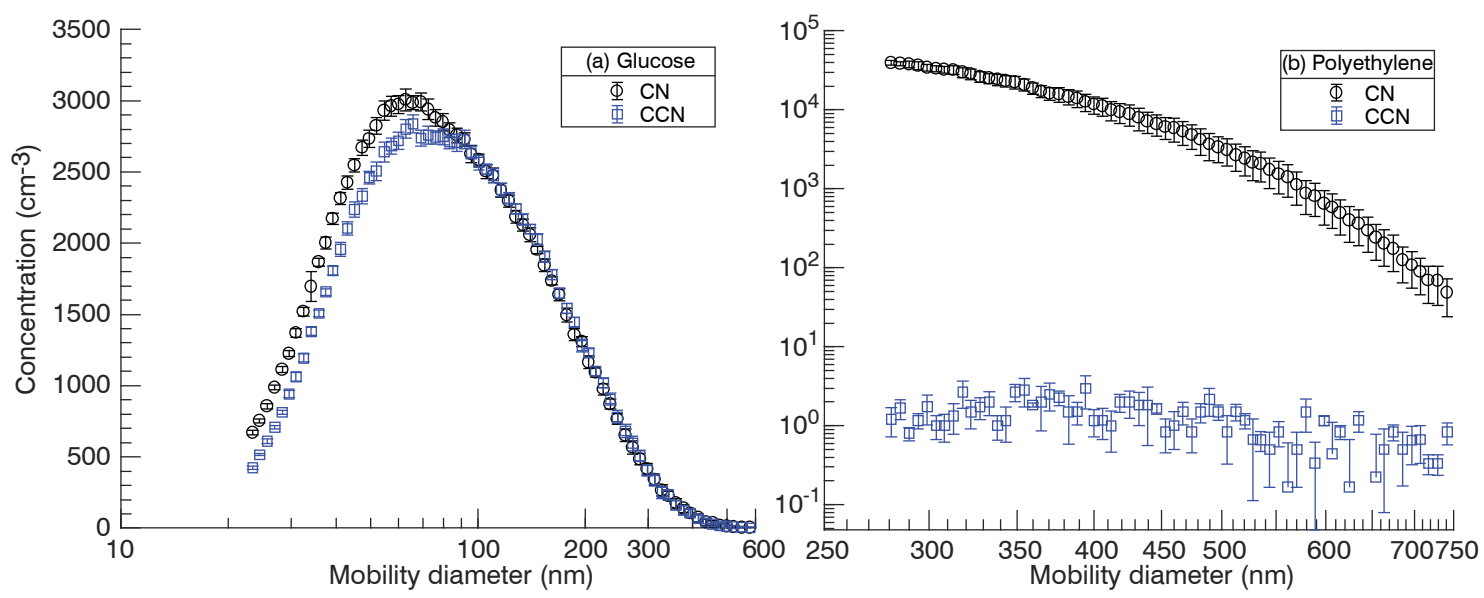

Figure S2. CCN experiments using (a) glucose and (b) polyethylene at $\mathrm{dT}=20 \mathrm{~K}$ and $\mathrm{CCNc}$ flow rate $1.0 \mathrm{~L} \mathrm{~min}^{-1}$. 


\section{Section S2. Data Tables}

Table S1a. Data for Figure 2a, left panel in the main text. Columns are midpoint temperature of the 3K bin, mean particle geometry factor of all particles within the bin, standard deviation of particle geometry factor, and relative humidity in the RDMA. The fitted logistic curve and uncertainty in the inferred parameters is given in Table 1 of the main text.

\begin{tabular}{|l|l|l|l|}
\hline $\mathrm{T}\left[{ }^{\circ} \mathrm{C}\right]$ & $\xi(-)$ & $\pm \xi(-)$ & $\mathrm{RH}(\%)$ \\
\hline 25.5 & 5.02 & 0.37 & 3.23 \\
\hline 28.5 & 4.13 & 0.32 & 3.21 \\
\hline 31.5 & 4.02 & 0.22 & 3.18 \\
\hline 34.5 & 4.71 & 0.75 & 3.13 \\
\hline 37.5 & 3.98 & 0.05 & 3.07 \\
\hline 40.5 & 3.96 & 0.16 & 3.02 \\
\hline 43.5 & 3.86 & 0.28 & 3.02 \\
\hline 46.5 & 3.80 & 0.15 & 3.07 \\
\hline 49.5 & 3.52 & 0.11 & 3.06 \\
\hline 52.5 & 3.46 & 0.32 & 2.82 \\
\hline 55.5 & 3.21 & 0.13 & 2.91 \\
\hline 58.5 & 3.10 & 0.10 & 3.01 \\
\hline 61.5 & 2.89 & 0.13 & 3.02 \\
\hline 64.5 & 2.90 & 0.22 & 2.99 \\
\hline 67.5 & 2.78 & 0.64 & 2.95 \\
\hline 70.5 & 2.29 & 0.25 & 2.85 \\
\hline 73.5 & 2.24 & 0.12 & 2.81 \\
\hline 76.5 & 1.83 & 0.06 & 2.82 \\
\hline 79.5 & 1.20 & 0.34 & 2.86 \\
\hline 82.5 & 1.08 & 0.11 & 2.85 \\
\hline 85.5 & 0.72 & 0.39 & 2.79 \\
\hline 88.5 & 1.02 & 0.01 & 2.74 \\
\hline 91.5 & 1.11 & 0.20 & 2.69 \\
\hline 94.5 & 0.86 & 0.59 & 2.68 \\
\hline & & & \\
\hline
\end{tabular}


Table S1b: Data for Figure 2b, left panel in the main text. Columns are midpoint temperature of the $3 \mathrm{~K}$ bin, mean particle geometry factor of all particles within the bin, standard deviation of particle geometry factor, and relative humidity in the RDMA. The fitted logistic curve and uncertainty in the inferred parameters is given in Table 1 of the main text.

\begin{tabular}{|c|c|c|c|}
\hline $\mathrm{T}\left[{ }^{\circ} \mathrm{C}\right]$ & $\xi(-)$ & $\pm \xi(-)$ & $\mathrm{RH}(\%)$ \\
\hline 25.5 & 4.20 & 0.72 & 4.19 \\
\hline 28.5 & 4.43 & 0.40 & 4.14 \\
\hline 31.5 & 3.80 & 0.42 & 4.06 \\
\hline 34.5 & 4.25 & 0.15 & 3.96 \\
\hline 37.5 & 4.45 & 0.49 & 3.86 \\
\hline 40.5 & 3.68 & 0.25 & 3.75 \\
\hline 43.5 & 4.26 & 0.27 & 3.62 \\
\hline 46.5 & 3.42 & 0.14 & 3.47 \\
\hline 49.5 & 3.66 & 0.65 & 3.30 \\
\hline 52.5 & 3.03 & 0.64 & 3.06 \\
\hline 55.5 & 3.15 & 0.62 & 2.84 \\
\hline 58.5 & 3.04 & 0.13 & 2.64 \\
\hline 61.5 & 2.79 & 0.02 & 2.43 \\
\hline 64.5 & 2.63 & 0.33 & 2.23 \\
\hline 67.5 & 2.19 & 0.27 & 2.03 \\
\hline 70.5 & 1.86 & 0.68 & 1.87 \\
\hline 73.5 & 1.21 & 0.21 & 1.59 \\
\hline 76.5 & 1.40 & 0.17 & 1.42 \\
\hline 79.5 & 0.90 & 0.26 & 1.34 \\
\hline 82.5 & 0.96 & 0.13 & 1.26 \\
\hline 85.5 & 0.91 & 0.11 & 1.20 \\
\hline 88.5 & 0.83 & 0.19 & 1.08 \\
\hline 91.5 & 1.08 & 0.62 & 1.01 \\
\hline 94.5 & 1.24 & 0.21 & 0.97 \\
\hline
\end{tabular}


Table S2a: Data for Figure 3a in the main text. Activation properties of ammonium sulfate monomers of $50 \mathrm{~nm}$ mode diameter over three different temperature gradients of the $\mathrm{CCNc}$ column corresponding to three different water supersaturation levels (\%).

\begin{tabular}{|c|c|c|c|c|c|c|}
\hline \multirow[t]{2}{*}{$\mathrm{Dp}(\mathrm{nm})$} & \multicolumn{2}{|c|}{$\mathrm{dT}=8 \mathrm{~K}$} & \multicolumn{2}{|c|}{$\mathrm{dT}=12 \mathrm{~K}$} & \multicolumn{2}{|c|}{$\mathrm{dT}=16 \mathrm{~K}$} \\
\hline & $\mathrm{CN}\left(\mathrm{cm}^{-3}\right)$ & $\mathrm{CCN}\left(\mathrm{cm}^{-3}\right)$ & $\mathrm{CN}\left(\mathrm{cm}^{-3}\right)$ & $\operatorname{CCN}\left(\mathrm{cm}^{-3}\right)$ & $\mathrm{CN}\left(\mathrm{cm}^{-3}\right)$ & $\operatorname{CCN}\left(\mathrm{cm}^{-3}\right)$ \\
\hline 117.8 & 64.1 & 51.1 & 62.4 & 52.2 & 62.9 & 51.6 \\
\hline 113.3 & 80.1 & 64.4 & 70.9 & 71.4 & 73.7 & 57.6 \\
\hline 109.6 & 82.3 & 77.2 & 79.9 & 78.3 & 80.9 & 69.8 \\
\hline 106.1 & 96.8 & 81.2 & 95.1 & 69.9 & 94.2 & 87.2 \\
\hline 102.1 & 112.0 & 93.0 & 104.5 & 107.6 & 101.6 & 93.5 \\
\hline 98.4 & 125.8 & 108.0 & 115.5 & 107.2 & 120.2 & 128.5 \\
\hline 94.8 & 141.3 & 120.3 & 136.4 & 111.1 & 133.3 & 125.3 \\
\hline 91.3 & 152.4 & 122.1 & 148.7 & 140.8 & 146.2 & 137.6 \\
\hline 88.4 & 172.3 & 145.7 & 162.2 & 149.2 & 150.2 & 126.4 \\
\hline 85.6 & 181.0 & 169.9 & 184.8 & 131.7 & 168.1 & 139.4 \\
\hline 82.6 & 210.6 & 155.6 & 205.9 & 206.5 & 201.2 & 202.2 \\
\hline 79.6 & 243.0 & 212.8 & 234.6 & 229.5 & 228.9 & 228.7 \\
\hline 76.8 & 281.4 & 247.6 & 274.1 & 195.7 & 272.6 & 249.8 \\
\hline 74.0 & 307.3 & 270.8 & 308.9 & 298.0 & 299.2 & 321.1 \\
\hline 71.4 & 334.7 & 245.9 & 322.1 & 332.8 & 308.5 & 310.5 \\
\hline 68.9 & 315.3 & 234.9 & 312.3 & 250.9 & 295.8 & 235.9 \\
\hline 66.5 & 274.6 & 173.6 & 263.4 & 240.8 & 263.7 & 250.4 \\
\hline 64.2 & 232.5 & 184.9 & 227.2 & 213.1 & 225.9 & 188.4 \\
\hline 61.9 & 210.3 & 146.7 & 210.8 & 162.4 & 210.1 & 147.6 \\
\hline 59.8 & 241.9 & 72.7 & 232.3 & 172.2 & 222.7 & 239.5 \\
\hline 57.7 & 329.0 & 26.4 & 320.6 & 266.4 & 316.6 & 289.8 \\
\hline 55.7 & 481.3 & 25.7 & 469.9 & 384.6 & 456.4 & 405.1 \\
\hline 53.8 & 632.3 & 25.8 & 630.9 & 463.9 & 619.8 & 605.0 \\
\hline 52.0 & 799.8 & 22.1 & 765.4 & 503.1 & 751.1 & 745.0 \\
\hline 50.2 & 874.5 & 24.9 & 845.2 & 484.0 & 852.0 & 835.0 \\
\hline 48.3 & 866.2 & 23.3 & 852.6 & 287.1 & 849.8 & 842.0 \\
\hline 46.4 & 749.3 & 17.2 & 754.9 & 198.9 & 751.0 & 660.7 \\
\hline 44.8 & 587.4 & 13.4 & 585.6 & 97.1 & 576.2 & 535.0 \\
\hline 43.3 & 400.9 & 4.8 & 389.1 & 39.4 & 379.9 & 334.5 \\
\hline 41.9 & 244.7 & 5.4 & 228.2 & 18.4 & 227.3 & 180.1 \\
\hline 40.4 & 132.7 & 0.0 & 124.2 & 9.1 & 120.7 & 65.7 \\
\hline 39.1 & 70.9 & 0.5 & 64.3 & 18.3 & 63.8 & 37.7 \\
\hline 37.6 & 46.1 & 1.3 & 40.8 & 19.0 & 40.4 & 34.4 \\
\hline 36.2 & 42.6 & 0.5 & 39.5 & 16.7 & 39.2 & 27.8 \\
\hline 35.0 & 39.1 & 0.0 & 39.4 & 15.1 & 35.6 & 31.0 \\
\hline 33.8 & 36.1 & 0.0 & 35.3 & 7.6 & 33.9 & 25.8 \\
\hline 32.7 & 30.3 & 0.0 & 28.7 & 4.3 & 27.3 & 16.1 \\
\hline 31.5 & 21.9 & 0.0 & 20.2 & 1.7 & 19.0 & 12.1 \\
\hline 30.3 & 12.0 & 0.5 & 11.1 & 0.5 & 9.6 & 7.0 \\
\hline
\end{tabular}


Table S2b: Data for Figure $3 b$ in the main text. Activation properties of AS-PE coalesced particles at 25 ${ }^{\circ} \mathrm{C}$, probably present in dimer morphology, over three different temperature gradients of the $\mathrm{CCNc}$ column corresponding to three different water supersaturation levels (\%).

\begin{tabular}{|c|c|c|c|c|c|c|}
\hline \multirow[t]{2}{*}{$\mathrm{Dp}(\mathrm{nm})$} & \multicolumn{2}{|c|}{$\mathrm{dT}=8 \mathrm{~K}$} & \multicolumn{2}{|c|}{$\mathrm{dT}=12 \mathrm{~K}$} & \multicolumn{2}{|c|}{$\mathrm{dT}=16 \mathrm{~K}$} \\
\hline & $\mathrm{CN}\left(\mathrm{cm}^{-3}\right)$ & $\mathrm{CCN}\left(\mathrm{cm}^{-3}\right)$ & $\mathrm{CN}\left(\mathrm{cm}^{-3}\right)$ & $\operatorname{CCN}\left(\mathrm{cm}^{-3}\right)$ & $\mathrm{CN}\left(\mathrm{cm}^{-3}\right)$ & $\operatorname{CCN}\left(\mathrm{cm}^{-3}\right)$ \\
\hline 117.8 & 0.3 & 0.6 & 0.2 & 1.1 & 0.4 & 0.0 \\
\hline 113.3 & 0.5 & 0.0 & 0.4 & 1.1 & 0.3 & 1.6 \\
\hline 109.6 & 0.4 & 0.7 & 0.7 & 0.0 & 0.4 & 0.0 \\
\hline 106.1 & 0.6 & 0.5 & 0.7 & 0.5 & 0.7 & 0.5 \\
\hline 102.1 & 0.9 & 0.5 & 1.2 & 1.6 & 0.9 & 2.1 \\
\hline 98.4 & 0.7 & 2.1 & 1.3 & 1.1 & 1.7 & 0.0 \\
\hline 94.8 & 1.7 & 0.5 & 1.8 & 1.6 & 1.3 & 0.5 \\
\hline 91.3 & 1.9 & 1.1 & 1.3 & 0.5 & 1.7 & 1.6 \\
\hline 88.4 & 1.4 & 0.7 & 1.5 & 2.9 & 1.1 & 0.7 \\
\hline 85.6 & 1.5 & 0.5 & 1.5 & 0.5 & 1.7 & 1.1 \\
\hline 82.6 & 2.3 & 0.5 & 2.1 & 1.1 & 1.7 & 0.7 \\
\hline 79.6 & 4.2 & 0.0 & 2.8 & 3.8 & 2.5 & 0.8 \\
\hline 76.8 & 8.2 & 0.0 & 5.9 & 4.8 & 5.8 & 12.3 \\
\hline 74.0 & 12.2 & 0.0 & 10.6 & 8.6 & 9.8 & 9.7 \\
\hline 71.4 & 19.1 & 1.1 & 17.1 & 8.1 & 15.6 & 15.1 \\
\hline 68.9 & 26.3 & 0.0 & 21.8 & 16.4 & 19.5 & 21.9 \\
\hline 66.5 & 30.5 & 0.0 & 23.9 & 15.0 & 26.4 & 24.0 \\
\hline 64.2 & 26.9 & 0.0 & 24.5 & 14.4 & 24.9 & 25.4 \\
\hline 61.9 & 25.3 & 0.0 & 21.4 & 8.5 & 23.4 & 24.2 \\
\hline 59.8 & 19.7 & 1.1 & 16.6 & 6.9 & 18.8 & 14.5 \\
\hline 57.7 & 14.0 & 0.0 & 14.2 & 1.6 & 12.3 & 5.9 \\
\hline 55.7 & 9.6 & 0.0 & 8.0 & 1.1 & 8.9 & 4.8 \\
\hline 53.8 & 6.1 & 0.0 & 4.9 & 1.1 & 5.8 & 4.8 \\
\hline 52.0 & 5.0 & 0.0 & 3.8 & 1.1 & 3.8 & 0.8 \\
\hline 50.2 & 3.5 & 0.0 & 3.4 & 1.6 & 3.2 & 0.8 \\
\hline 48.3 & 3.8 & 0.0 & 3.2 & 0.9 & 2.6 & 2.6 \\
\hline 46.4 & 3.2 & 0.0 & 2.7 & 2.2 & 3.0 & 1.6 \\
\hline 44.8 & 2.8 & 0.0 & 2.5 & 0.0 & 2.3 & 2.2 \\
\hline 43.3 & 2.3 & 0.0 & 2.2 & 0.0 & 2.5 & 4.8 \\
\hline 41.9 & 1.8 & 0.0 & 1.7 & 0.5 & 1.0 & 1.1 \\
\hline 40.4 & 0.7 & 0.0 & 0.9 & 0.0 & 1.5 & 1.1 \\
\hline 39.1 & 1.0 & 0.0 & 0.4 & 0.0 & 0.6 & 0.5 \\
\hline 37.6 & 0.3 & 0.0 & 0.2 & 0.0 & 0.5 & 0.0 \\
\hline 36.2 & 0.3 & 0.0 & 0.1 & 0.5 & 0.1 & 0.0 \\
\hline 35.0 & 0.2 & 0.0 & 0.1 & 0.0 & 0.1 & 0.0 \\
\hline 33.8 & 0.1 & 0.0 & 0.0 & 0.5 & 0.1 & 0.0 \\
\hline 32.7 & 0.0 & 0.0 & 0.1 & 0.0 & 0.1 & 0.0 \\
\hline 31.5 & 0.0 & 0.0 & 0.0 & 0.0 & 0.0 & 0.0 \\
\hline 30.3 & 0.0 & 0.0 & 0.0 & 0.5 & 0.0 & 0.0 \\
\hline
\end{tabular}


Table S2c: Data for Figure 3c in the main text. Activation properties of AS-PE coagulated particles at $95{ }^{\circ} \mathrm{C}$, most likely present in core-shell morphology, over three different temperature gradients of the $\mathrm{CCNc}$ column corresponding to three different water supersaturation levels (\%).

\begin{tabular}{|r|r|r|r|r|r|r|}
\hline \multirow{2}{*}{$\mathrm{Dp}(\mathrm{nm})$} & \multicolumn{2}{|c|}{$\mathrm{dT}=8 \mathrm{~K}$} & \multicolumn{2}{c|}{$\mathrm{dT}=12 \mathrm{~K}$} & \multicolumn{2}{c|}{$\mathrm{dT}=16 \mathrm{~K}$} \\
\cline { 2 - 7 } & $\mathrm{CN}\left(\mathrm{cm}^{-3}\right)$ & $\mathrm{CCN}\left(\mathrm{cm}^{-3}\right)$ & $\mathrm{CN}\left(\mathrm{cm}^{-3}\right)$ & $\mathrm{CCN}\left(\mathrm{cm}^{-3}\right)$ & $\mathrm{CN}\left(\mathrm{cm}^{-3}\right)$ & $\mathrm{CCN}\left(\mathrm{cm}^{-3}\right)$ \\
\hline 117.8 & 0.0 & 0.0 & 0.1 & 0.5 & 0.1 & 0.0 \\
\hline 113.3 & 0.3 & 0.0 & 0.2 & 0.0 & 0.1 & 0.0 \\
\hline 109.6 & 0.1 & 0.0 & 0.2 & 0.0 & 0.2 & 0.0 \\
\hline 106.1 & 0.1 & 0.0 & 0.1 & 0.6 & 0.4 & 0.0 \\
\hline 102.1 & 0.2 & 0.0 & 0.3 & 1.1 & 0.5 & 0.0 \\
\hline 98.4 & 0.6 & 1.1 & 0.5 & 0.0 & 0.4 & 1.1 \\
\hline 94.8 & 0.7 & 0.5 & 0.9 & 1.1 & 0.7 & 0.0 \\
\hline 91.3 & 0.8 & 0.0 & 0.7 & 1.6 & 0.7 & 2.1 \\
\hline 88.4 & 0.5 & 0.0 & 1.0 & 0.0 & 0.9 & 0.7 \\
\hline 85.6 & 0.7 & 1.1 & 1.2 & 0.0 & 1.0 & 1.6 \\
\hline 82.6 & 0.7 & 1.6 & 0.9 & 1.5 & 0.9 & 1.1 \\
\hline 79.6 & 0.9 & 0.5 & 1.0 & 2.3 & 1.3 & 0.0 \\
\hline 76.8 & 1.1 & 1.1 & 1.5 & 1.6 & 1.9 & 0.5 \\
\hline 74.0 & 2.3 & 0.0 & 2.6 & 2.1 & 3.1 & 5.4 \\
\hline 71.4 & 3.2 & 0.0 & 4.8 & 3.8 & 5.7 & 4.3 \\
\hline 68.9 & 6.0 & 0.0 & 8.3 & 5.3 & 7.1 & 6.5 \\
\hline 66.5 & 10.5 & 0.0 & 12.0 & 11.2 & 12.4 & 13.2 \\
\hline 64.2 & 14.2 & 0.0 & 14.1 & 10.3 & 15.4 & 18.2 \\
\hline 61.9 & 17.7 & 0.0 & 15.5 & 10.1 & 16.9 & 16.5 \\
\hline 59.8 & 18.5 & 0.0 & 17.5 & 8.0 & 17.9 & 16.1 \\
\hline 57.7 & 17.2 & 0.0 & 14.1 & 5.9 & 15.0 & 17.2 \\
\hline 55.7 & 15.5 & 0.0 & 13.0 & 4.0 & 13.4 & 12.3 \\
\hline 53.8 & 10.6 & 0.0 & 9.2 & 0.0 & 10.8 & 7.2 \\
\hline 52.0 & 8.0 & 0.0 & 6.0 & 1.1 & 7.1 & 4.2 \\
\hline 50.2 & 5.3 & 0.0 & 4.4 & 0.0 & 4.7 & 2.7 \\
\hline 48.3 & 3.3 & 0.0 & 2.6 & 0.9 & 2.5 & 0.4 \\
\hline 46.4 & 1.7 & 0.0 & 2.1 & 0.5 & 2.7 & 0.0 \\
\hline 44.8 & 1.9 & 0.0 & 1.5 & 1.1 & 1.5 & 1.6 \\
\hline 43.3 & 1.6 & 0.0 & 1.2 & 0.0 & 1.6 & 0.5 \\
\hline 41.9 & 0.9 & 0.0 & 0.8 & 0.5 & 1.1 & 0.0 \\
\hline 40.4 & 1.4 & 0.0 & 0.8 & 0.0 & 1.0 & 1.1 \\
\hline 39.1 & 1.0 & 0.0 & 0.5 & 0.5 & 0.8 & 1.1 \\
\hline 37.6 & 0.8 & 0.0 & 0.6 & 0.4 & 0.3 & 0.0 \\
\hline 36.2 & 0.6 & 0.0 & 0.2 & 0.0 & 0.3 & 0.0 \\
\hline 35.0 & 0.3 & 0.0 & 0.4 & 0.0 & 0.2 & 0.0 \\
\hline 33.8 & 0.0 & 0.0 & 0.1 & 0.0 & 0.1 & 0.0 \\
\hline 32.7 & 0.0 & 0.0 & 0.1 & 0.0 & 0.1 & 0.0 \\
\hline 31.5 & 0.0 & 0.0 & 0.0 & 0.0 & 0.1 & 0.3 \\
\hline 30.3 & 0.0 & 0.0 & 0.0 & 0.0 & 0.0 & 0.0 \\
\hline & & & & & & \\
\hline
\end{tabular}


Table S3: Data for Figure 4 in the main text. Activated Fraction (mean \pm standard deviation) of AS Monomers $(50 \mathrm{~nm})$, AS $(50 \mathrm{~nm})+\mathrm{PE}(50 \mathrm{~nm})$ coalesced particles, conditioned at $25^{\circ} \mathrm{C}$, to be present in dimer morphology and AS $(50 \mathrm{~nm})+\mathrm{PE}(50 \mathrm{~nm})$ coagulated particles, conditioned at $95{ }^{\circ} \mathrm{C}$, to be present in core-shell morphology over different water supersaturation (\%) levels.

\begin{tabular}{|c|c|c|c|}
\hline $\begin{array}{c}\text { Water } \\
\text { supersaturation } \\
(\%)\end{array}$ & AS Monomers $(50 \mathrm{~nm})$ & $\begin{array}{c}\mathrm{AS}(50 \mathrm{~nm})+\mathrm{PE}(50 \mathrm{~nm}) \\
\text { coalesced particles at 25 }{ }^{\circ} \mathrm{C} \\
\text { in Dimer morphology }\end{array}$ & $\begin{array}{c}\text { AS }(50 \mathrm{~nm})+\mathrm{PE}(50 \mathrm{~nm}) \\
\text { coagulated particles at } 95{ }^{\circ} \mathrm{C} \text { in } \\
\text { Core-Shell morphology }\end{array}$ \\
\hline 0.30 & $0.01 \pm 0.01$ & $0.00 \pm 0.00$ & $0.00 \pm 0.00$ \\
\hline 0.34 & $0.03 \pm 0.00$ & $0.00 \pm 0.00$ & $0.01 \pm 0.01$ \\
\hline 0.38 & $0.04 \pm 0.01$ & $0.02 \pm 0.02$ & $0.01 \pm 0.01$ \\
\hline 0.42 & $0.17 \pm 0.10$ & $0.03 \pm 0.03$ & $0.18 \pm 0.10$ \\
\hline 0.46 & $0.39 \pm 0.06$ & $0.19 \pm 0.07$ & $0.55 \pm 0.12$ \\
\hline 0.50 & $0.76 \pm 0.02$ & $0.51 \pm 0.08$ & $0.68 \pm 0.11$ \\
\hline 0.54 & $1.00 \pm 0.10$ & $0.65 \pm 0.08$ & $0.85 \pm 0.02$ \\
\hline 0.58 & $1.01 \pm 0.02$ & $0.89 \pm 0.08$ & $0.80 \pm 0.06$ \\
\hline 0.62 & $1.00 \pm 0.01$ & $1.02 \pm 0.07$ & $0.87 \pm 0.10$ \\
\hline 0.66 & $0.87 \pm 0.11$ & 0.03 \\
\hline
\end{tabular}


Table S4: Data for Figure 5 in the main text. Activated Fraction (mean \pm standard deviation) of AS Monomers along with AS-PE coagulated particles, formed by the coalescence of $50 \mathrm{~nm}$ mode diameter AS and $50 \mathrm{~nm}, 60 \mathrm{~nm}, 80 \mathrm{~nm}, 100 \mathrm{~nm}$ and $120 \mathrm{~nm}$ mode diameter PE particles, conditioned at $95^{\circ} \mathrm{C}$ to be present in core-shell morphology.

\begin{tabular}{|c|c|c|c|c|c|c|}
\hline $\begin{array}{c}\text { Water } \\
\text { supersaturation } \\
(\%)\end{array}$ & $\begin{array}{c}\text { AS } \\
\text { Monomers } \\
(50 \mathrm{~nm}) \\
\end{array}$ & $\begin{array}{c}\text { AS }(50 \mathrm{~nm}) \\
+\mathrm{PE}(50 \\
\mathrm{nm})\end{array}$ & $\begin{array}{c}\text { AS }(50 \mathrm{~nm}) \\
+\mathrm{PE}(60 \\
\mathrm{nm})\end{array}$ & $\begin{array}{c}\text { AS }(50 \mathrm{~nm})+ \\
\text { PE }(80 \mathrm{~nm})\end{array}$ & $\begin{array}{l}\text { AS }(50 \mathrm{~nm})+ \\
\text { PE }(100 \mathrm{~nm})\end{array}$ & $\begin{array}{l}\text { AS }(50 \mathrm{~nm})+ \\
\operatorname{PE}(120 \mathrm{~nm})\end{array}$ \\
\hline 0.30 & $0.01 \pm 0.01$ & $0.00 \pm 0.00$ & - & - & - & - \\
\hline 0.34 & $0.02 \pm 0.00$ & $0.01 \pm 0.01$ & $0.01 \pm 0.00$ & - & - & - \\
\hline 0.38 & $0.03 \pm 0.01$ & $0.01 \pm 0.01$ & $0.00 \pm 0.00$ & - & - & - \\
\hline 0.42 & $0.04 \pm 0.01$ & $0.03 \pm 0.03$ & $0.01 \pm 0.00$ & - & - & - \\
\hline 0.46 & $0.17 \pm 0.10$ & $0.18 \pm 0.10$ & $0.21 \pm 0.03$ & $0.16 \pm 0.00$ & $0.16 \pm 0.05$ & $0.17 \pm 0.02$ \\
\hline 0.50 & $0.39 \pm 0.06$ & $0.55 \pm 0.12$ & $0.40 \pm 0.09$ & $0.44 \pm 0.06$ & $0.51 \pm 0.02$ & $0.41 \pm 0.04$ \\
\hline 0.54 & $0.76 \pm 0.02$ & $0.68 \pm 0.11$ & $0.65 \pm 0.14$ & $0.63 \pm 0.01$ & $0.64 \pm 0.04$ & $0.63 \pm 0.04$ \\
\hline 0.58 & $1.00 \pm 0.10$ & $0.85 \pm 0.02$ & $0.88 \pm 0.02$ & $0.83 \pm 0.15$ & $0.75 \pm 0.04$ & $0.68 \pm 0.09$ \\
\hline 0.62 & $1.01 \pm 0.02$ & $0.80 \pm 0.06$ & $0.98 \pm 0.00$ & $0.83 \pm 0.02$ & $0.78 \pm 0.04$ & $0.80 \pm 0.02$ \\
\hline 0.66 & $1.00 \pm 0.01$ & $0.87 \pm 0.10$ & - & - & - & - \\
\hline
\end{tabular}


Table S5: Data for Figure S1. Data-set used to construct dT(K) vs SS(\%) calibration curve using AS particles in DMT CCNc with a total flow rate of $0.3 \mathrm{~L} \mathrm{~min}^{-1}$.

\begin{tabular}{|r|r|c|}
\hline \multicolumn{1}{|c|}{$\mathrm{dT}(\mathrm{K})$} & \multicolumn{1}{|c|}{$\mathrm{Dd}_{50}(\mathrm{~nm})$} & $\mathrm{SS}(\%)$ \\
\hline 5 & $90.4 \pm 0.6$ & $0.17 \pm 0.00$ \\
\hline 6 & $71.7 \pm 1.2$ & $0.25 \pm 0.01$ \\
\hline 7 & $62.6 \pm 2.1$ & $0.30 \pm 0.02$ \\
\hline 8 & $57.4 \pm 1.5$ & $0.35 \pm 0.01$ \\
\hline 9 & $53.8 \pm 1.2$ & $0.38 \pm 0.01$ \\
\hline 10 & $50.6 \pm 1.5$ & $0.42 \pm 0.02$ \\
\hline 11 & $47.3 \pm 0.0$ & $0.47 \pm 0.00$ \\
\hline 12 & $44.4 \pm 1.0$ & $0.52 \pm 0.02$ \\
\hline 13 & $42.7 \pm 0.9$ & $0.55 \pm 0.02$ \\
\hline 14 & $40.9 \pm 0.5$ & $0.59 \pm 0.01$ \\
\hline 15 & $39.3 \pm 0.5$ & $0.63 \pm 0.01$ \\
\hline 16 & $38.2 \pm 0.5$ & $0.66 \pm 0.01$ \\
\hline 17 & $36.9 \pm 0.2$ & $0.69 \pm 0.01$ \\
\hline 18 & $35.8 \pm 0.3$ & $0.73 \pm 0.01$ \\
\hline 19 & $35.1 \pm 0.3$ & $0.75 \pm 0.01$ \\
\hline
\end{tabular}


Table S6: Data for Figure S2a. Concentrations of Glucose particles as $\mathrm{CN}$ and as $\mathrm{CCN}$ are mean of nine different scans along with associated standard deviations.

\begin{tabular}{|c|c|c|c|c|c|}
\hline $\mathrm{Dp}(\mathrm{nm})$ & $\mathrm{CN}\left(\mathrm{cm}^{-3}\right)$ & $\operatorname{CCN}\left(\mathrm{cm}^{-3}\right)$ & $\mathrm{Dp}(\mathrm{nm})$ & $\mathrm{CN}\left(\mathrm{cm}^{-3}\right)$ & $\operatorname{CCN}\left(\mathrm{cm}^{-3}\right)$ \\
\hline 579.9 & $6.7 \pm 5.1$ & $5.8 \pm 3.4$ & 116.2 & $2355.3 \pm 48.2$ & $2373.9 \pm 45.8$ \\
\hline 549.7 & $12.0 \pm 7.1$ & $10.2 \pm 5.0$ & 110.5 & $2441.4 \pm 50.4$ & $2439.4 \pm 47.8$ \\
\hline 521.4 & $18.9 \pm 9.3$ & $18.4 \pm 8.4$ & 105.7 & $2490.3 \pm 47.8$ & $2479.3 \pm 38.9$ \\
\hline 498.3 & $28.9 \pm 12.5$ & $24.9 \pm 10.7$ & 100.6 & $2554.7 \pm 41.7$ & $2550.2 \pm 50.5$ \\
\hline 476.5 & $39.4 \pm 15.8$ & $37.6 \pm 17.5$ & 95.8 & $2623.1 \pm 62.1$ & $2568.9 \pm 44.5$ \\
\hline 455.9 & $54.3 \pm 17.5$ & $50.6 \pm 18.1$ & 91.8 & $2680.8 \pm 45.4$ & $2609.6 \pm 64.9$ \\
\hline 436.2 & $73.8 \pm 23.8$ & $68.3 \pm 19.6$ & 87.4 & $2726.9 \pm 49.8$ & $2667.5 \pm 60.9$ \\
\hline 414.6 & $101.1 \pm 27.8$ & $95.2 \pm 23.1$ & 83.3 & $2769.2 \pm 50.8$ & $2675.9 \pm 50.7$ \\
\hline 394.2 & $136.0 \pm 26.6$ & $132.1 \pm 28.2$ & 79.8 & $2816.7 \pm 58.9$ & $2721.1 \pm 56.3$ \\
\hline 377.6 & $173.6 \pm 28.8$ & $164.4 \pm 29.5$ & 76.1 & $2858.7 \pm 57.0$ & $2718.2 \pm 46.7$ \\
\hline 359.4 & $220.9 \pm 32.7$ & $217.0 \pm 34.0$ & 72.6 & $2873.9 \pm 74.2$ & $2741.5 \pm 62.5$ \\
\hline 342.2 & $275.3 \pm 32.1$ & $275.2 \pm 34.6$ & 69.3 & $2909.1 \pm 62.0$ & $2741.2 \pm 55.8$ \\
\hline 328.2 & $326.0 \pm 39.8$ & $328.7 \pm 42.4$ & 65.8 & $2930.9 \pm 47.8$ & $2740.8 \pm 63.7$ \\
\hline 312.8 & $401.5 \pm 39.8$ & $398.8 \pm 40.9$ & 62.8 & $2925.0 \pm 76.9$ & $2698.1 \pm 65.0$ \\
\hline 298.2 & $471.1 \pm 40.6$ & $473.2 \pm 41.0$ & 59.9 & $2918.2 \pm 62.0$ & $2684.4 \pm 62.0$ \\
\hline 284.5 & $545.4 \pm 40.4$ & $558.6 \pm 40.7$ & 57.2 & $2885.8 \pm 70.5$ & $2632.6 \pm 55.4$ \\
\hline 271.5 & $630.6 \pm 45.7$ & $649.9 \pm 50.2$ & 54.7 & $2835.1 \pm 66.4$ & $2573.4 \pm 69.6$ \\
\hline 259.3 & $727.1 \pm 44.9$ & $740.9 \pm 48.4$ & 51.9 & $2773.3 \pm 59.0$ & $2478.8 \pm 64.7$ \\
\hline 246.1 & $833.0 \pm 37.0$ & $846.2 \pm 44.1$ & 49.6 & $2700.3 \pm 60.6$ & $2386.9 \pm 45.3$ \\
\hline 235.2 & $927.2 \pm 35.2$ & $939.5 \pm 40.5$ & 47.4 & $2604.0 \pm 51.6$ & $2294.8 \pm 52.5$ \\
\hline 224.9 & $1034.0 \pm 38.4$ & $1049.9 \pm 32.2$ & 45.1 & $2495.8 \pm 44.8$ & $2164.8 \pm 54.2$ \\
\hline 213.8 & $1138.1 \pm 33.8$ & $1153.4 \pm 33.5$ & 43.1 & $2396.7 \pm 46.4$ & $2044.0 \pm 45.8$ \\
\hline 204.6 & $1227.0 \pm 44.2$ & $1267.2 \pm 31.1$ & 41.2 & $2268.9 \pm 41.5$ & $1929.4 \pm 47.6$ \\
\hline 195.9 & $1338.9 \pm 35.9$ & $1367.1 \pm 44.2$ & 39.2 & $2130.4 \pm 40.5$ & $1765.8 \pm 29.8$ \\
\hline 186.5 & $1437.8 \pm 42.0$ & $1477.4 \pm 32.4$ & 37.5 & $1983.9 \pm 41.6$ & $1642.6 \pm 24.1$ \\
\hline 177.6 & $1546.1 \pm 49.0$ & $1588.7 \pm 34.0$ & 35.8 & $1837.6 \pm 27.6$ & $1488.1 \pm 22.7$ \\
\hline 169.2 & $1660.8 \pm 40.9$ & $1693.6 \pm 37.0$ & 34.1 & $1644.7 \pm 102.9$ & $1338.7 \pm 28.0$ \\
\hline 161.4 & $1754.4 \pm 29.0$ & $1793.3 \pm 34.2$ & 32.5 & $1505.3 \pm 31.5$ & $1189.2 \pm 27.8$ \\
\hline 153.9 & $1862.5 \pm 42.7$ & $1906.4 \pm 38.3$ & 30.9 & $1361.6 \pm 29.5$ & $1040.9 \pm 31.0$ \\
\hline 146.8 & $1955.3 \pm 23.8$ & $1988.3 \pm 38.4$ & 29.6 & $1218.1 \pm 22.7$ & $924.5 \pm 26.5$ \\
\hline 140.2 & $2041.1 \pm 48.9$ & $2070.2 \pm 33.7$ & 28.3 & $1097.7 \pm 20.8$ & $817.4 \pm 15.6$ \\
\hline 133.9 & $2119.4 \pm 40.5$ & $2164.3 \pm 39.8$ & 27.0 & $973.8 \pm 18.3$ & $704.6 \pm 10.7$ \\
\hline 127.9 & $2191.4 \pm 42.2$ & $2235.3 \pm 36.2$ & 25.7 & $845.4 \pm 19.2$ & $598.9 \pm 16.3$ \\
\hline \multirow[t]{2}{*}{122.2} & $2279.2 \pm 40.7$ & $2286.8 \pm 40.3$ & 24.4 & $730.2 \pm 15.5$ & $517.9 \pm 7.0$ \\
\hline & & & 23.4 & $642.0 \pm 15.2$ & $438.8 \pm 11.0$ \\
\hline
\end{tabular}


Table S7: Data for Figure S2b. Concentrations of PE particles as CN and as CCN are mean of three different scans along with associated standard deviations.

\begin{tabular}{|c|c|c|c|c|c|}
\hline $\mathrm{Dp}(\mathrm{nm})$ & $\mathrm{CN}\left(\mathrm{cm}^{-3}\right)$ & $\operatorname{CCN}\left(\mathrm{cm}^{-3}\right)$ & $\mathrm{Dp}(\mathrm{nm})$ & $\mathrm{CN}\left(\mathrm{cm}^{-3}\right)$ & $\operatorname{CCN}\left(\mathrm{cm}^{-3}\right)$ \\
\hline 741.3 & $21.6 \pm 24.3$ & $0.6 \pm 0.3$ & 453.3 & $4771.7 \pm 1911.6$ & $1.3 \pm 0.4$ \\
\hline 729.2 & $29.9 \pm 35.0$ & $0.4 \pm 0.1$ & 446.4 & $5180.8 \pm 1965.3$ & $1.7 \pm 0.3$ \\
\hline 717.4 & $32.0 \pm 34.4$ & $0.4 \pm 0.1$ & 439.4 & $5699.5 \pm 2151.9$ & $1.9 \pm 1.3$ \\
\hline 707.2 & $44.7 \pm 43.5$ & $0.6 \pm 0.3$ & 432.8 & $6230.8 \pm 2491.2$ & $1.0 \pm 0.8$ \\
\hline 697.1 & $54.3 \pm 52.2$ & $0.7 \pm 0.3$ & 425.8 & $6971.3 \pm 2690.3$ & $1.9 \pm 0.7$ \\
\hline 685.9 & $63.6 \pm 60.1$ & $0.5 \pm 0.3$ & 418.7 & $7525.0 \pm 2565.4$ & $1.4 \pm 0.5$ \\
\hline 674.8 & $83.5 \pm 85.5$ & $0.9 \pm 0.2$ & 412.4 & $8178.3 \pm 2739.9$ & $1.2 \pm 0.5$ \\
\hline 664.0 & $98.3 \pm 98.7$ & $0.6 \pm 0.4$ & 406.3 & $9225.8 \pm 2892.4$ & $1.7 \pm 0.5$ \\
\hline 654.6 & $123.9 \pm 115.4$ & $0.7 \pm 0.6$ & 400.3 & $9879.2 \pm 2921.6$ & $1.5 \pm 0.4$ \\
\hline 645.5 & $160.3 \pm 138.8$ & $0.3 \pm 0.4$ & 394.4 & $10470.8 \pm 3104.3$ & $2.1 \pm 1.4$ \\
\hline 635.1 & $199.8 \pm 172.6$ & $0.8 \pm 0.3$ & 388.6 & $11424.2 \pm 3252.3$ & $1.8 \pm 0.5$ \\
\hline 625.0 & $222.5 \pm 190.9$ & $0.7 \pm 0.5$ & 382.9 & $12409.2 \pm 3200.4$ & $1.7 \pm 0.9$ \\
\hline 615.0 & $272.2 \pm 235.1$ & $0.7 \pm 0.2$ & 376.6 & $13417.3 \pm 3280.4$ & $1.8 \pm 0.5$ \\
\hline 606.4 & $329.4 \pm 271.8$ & $0.6 \pm 0.7$ & 370.4 & $13991.7 \pm 2969.7$ & $1.9 \pm 1.0$ \\
\hline 598.0 & $370.7 \pm 297.1$ & $1.2 \pm 0.2$ & 365.0 & $15041.7 \pm 2954.1$ & $1.6 \pm 1.1$ \\
\hline 588.6 & $483.7 \pm 364.7$ & $0.7 \pm 0.3$ & 9.7 & $16133.3 \pm 3137.1$ & $1.8 \pm 0.1$ \\
\hline 579.3 & $569.1 \pm 402.3$ & $0.8 \pm 0.7$ & 354.5 & $17383.3 \pm 3599.7$ & $1.9 \pm 1.1$ \\
\hline 570.2 & $689.3 \pm 502.9$ & $0.5 \pm 0.3$ & 348.8 & $18520.0 \pm 4031.8$ & $2.0 \pm 0.7$ \\
\hline 561.2 & $814.5 \pm 625.6$ & $0.7 \pm 0.4$ & 343.1 & $19558.3 \pm 3881.4$ & $1.4 \pm 0.5$ \\
\hline 552.4 & $934.9 \pm 680.9$ & $0.7 \pm 0.3$ & 338.2 & $20841.7 \pm 4007.1$ & $0.9 \pm 0.3$ \\
\hline 543.8 & $1084.7 \pm 737.2$ & $0.8 \pm 0.3$ & 333.4 & $22150.0 \pm 3814.2$ & $1.3 \pm 0.7$ \\
\hline 535.3 & $1325.1 \pm 866.5$ & $0.6 \pm 0.2$ & 328.0 & $23253.3 \pm 3953.8$ & $1.2 \pm 0.5$ \\
\hline 528.0 & $1415.9 \pm 888.0$ & $0.7 \pm 0.6$ & 322.8 & $25083.3 \pm 4424.5$ & $1.2 \pm 0.6$ \\
\hline 520.9 & $1583.3 \pm 960.2$ & $0.9 \pm 0.3$ & 318.2 & $26558.3 \pm 4189.6$ & $1.7 \pm 1.0$ \\
\hline 512.8 & $1841.4 \pm 1003.0$ & $1.1 \pm 0.3$ & 313.2 & $28320.0 \pm 4073.5$ & $0.9 \pm 0.6$ \\
\hline 504.9 & $2190.0 \pm 1186.1$ & $0.8 \pm 0.5$ & 308.2 & $29408.3 \pm 3280.1$ & $0.8 \pm 0.4$ \\
\hline 497.2 & $2388.6 \pm 1214.8$ & $1.2 \pm 0.3$ & 303.9 & $30858.3 \pm 2982.9$ & $0.7 \pm 0.3$ \\
\hline 489.5 & $2639.2 \pm 1377.3$ & $1.3 \pm 0.8$ & 299.2 & $32486.7 \pm 3403.1$ & $0.9 \pm 0.7$ \\
\hline 482.0 & $3048.3 \pm 1522.7$ & $1.1 \pm 0.4$ & 294.5 & $34066.7 \pm 4043.4$ & $1.4 \pm 0.3$ \\
\hline 474.6 & $3538.3 \pm 1691.0$ & $0.6 \pm 0.4$ & 289.9 & $35760.0 \pm 3412.2$ & $0.6 \pm 0.2$ \\
\hline 467.4 & $3951.7 \pm 1802.4$ & $1.2 \pm 0.5$ & 285.4 & $37091.7 \pm 2552.3$ & $1.1 \pm 0.5$ \\
\hline 460.3 & $4412.5 \pm 1947.5$ & $1.1 \pm 0.5$ & 281.0 & $38000.0 \pm 2529.1$ & $1.3 \pm 0.5$ \\
\hline
\end{tabular}


Table S8: Mobility diameter (nm) of AS and PE monomers along with AS-PE coagulated particles at two different conditioner temperatures $\left({ }^{\circ} \mathrm{C}\right)$ over which AS-PE coagulated particles are expected to be present in Dimer morphology and Core-shell morphology, respectively. Here ' $n$ ' gives number scans averaged.

\begin{tabular}{|l|l|l|l|l|}
\hline $\begin{array}{l}\text { Conditioner } \\
\text { Temperature }\left({ }^{\circ} \mathrm{C}\right)\end{array}$ & \multicolumn{2}{|l|}{ Mobility diameter $(\mathrm{nm})$} & Expected Morphology \\
\cline { 2 - 5 } & AS monomer & PE monomer & AS-PE Coagulated & \\
\hline 25 & $49.29 \pm 0.38$ & $49.76 \pm 0.25$ & $65.09 \pm 0.39$ & Dimer morphology \\
& $(n=3)$ & $(n=3)$ & $(n=29)$ & Core-shell morphology \\
\hline 95 & $49.64 \pm 0.26$ & $50.13 \pm 0.74$ & $60.67 \pm 0.54$ & \\
$(n=3)$ & $(n=3)$ & $(n=30)$ & \\
\hline
\end{tabular}

